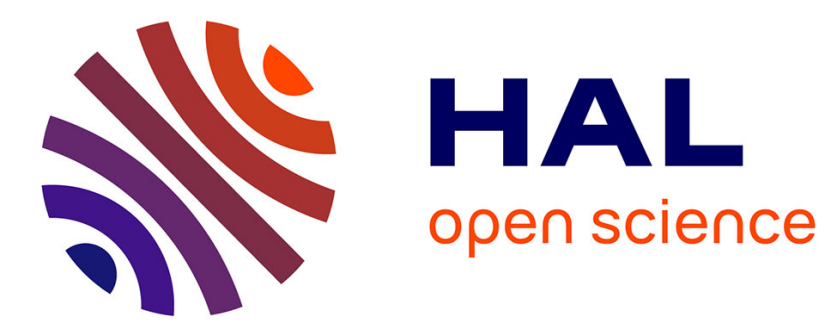

\title{
Identification of the ferromagnetic hysteresis simulation parameters using classic non-destructive testing equipment
}

\author{
S. Zhang, Benjamin Ducharne, S. Takeda, G. Sebald, T. Uchimoto
}

\section{- To cite this version:}

S. Zhang, Benjamin Ducharne, S. Takeda, G. Sebald, T. Uchimoto. Identification of the ferromagnetic hysteresis simulation parameters using classic non-destructive testing equipment. Journal of Magnetism and Magnetic Materials, 2021, pp.167971. 10.1016/j.jmmm.2021.167971 . hal-03193917

\section{HAL Id: hal-03193917 https://hal.science/hal-03193917}

Submitted on 9 Apr 2021

HAL is a multi-disciplinary open access archive for the deposit and dissemination of scientific research documents, whether they are published or not. The documents may come from teaching and research institutions in France or abroad, or from public or private research centers.
L'archive ouverte pluridisciplinaire HAL, est destinée au dépôt et à la diffusion de documents scientifiques de niveau recherche, publiés ou non, émanant des établissements d'enseignement et de recherche français ou étrangers, des laboratoires publics ou privés. 


\title{
Identification of the ferromagnetic hysteresis simulation parameters using classic non-destructive testing equipment.
}

\author{
S. Zhang ${ }^{1}$, B. Ducharne ${ }^{2,3}$, S. Takeda ${ }^{1}$, G. Sebald ${ }^{2}$, T. Uchimoto ${ }^{1,2}$
}

1. Tohoku University, Institute of fluid science IFS, Sendai, Japan.

2. ELyTMaX UMI 3757, CNRS - Université de Lyon - Tohoku University, International Joint Unit, Tohoku University, Sendai, Japan.

3. LGEF, INSA Lyon, Villeurbanne, France. 


\section{Abstract:}

Electromagnetic non-destructive controls are performed daily by steel manufacturers and steel user companies. Many methods exist, including Eddy Current Testing (ECT), Magnetic Particle Inspection (MPI), Magnetic Incremental Permeability (MIP), Magnetic Barkhausen noise (MBN) ... In this domain, progresses are constant, however there are limitations and accurate simulation tools able to improve the methods efficiency are still expected. Most of steels are ferromagnetic and characterized by a strong nonlinear hysteretic magnetic behavior. A precise taken into account of hysteresis is mandatory in the development of useful simulation tools. The hysteresis standard of characterization imposes restrictive conditions (geometrical, electrical ...) and most of Non-Destructive Testing (NDT) industrial end-users face difficulties to obtain these inevitable experimental results. In this manuscript, we describe an alternative method to adjust the hysteresis simulation parameters based on experimental results coming from classic NDT industrial equipment. The acquisition of a flat coil impedance in an ECT configuration and under the superimposition of a quasi-static magnetic excitation (MPI equipment) is performed to plot the tested specimen MIP signature. The Dodd \& Deeds (D\&D) analytical solution for an ECT flat coil is used to return the corresponding permeability. An optimization process based on a modified Jiles-Atherton (J-A) model is run to extract a suitable combination of hysteresis parameters. Finally, the good comparison with an experimental $B(H)$ characterization is worth as a validation of the method.

\section{Keywords:}

Ferromagnetic hysteresis simulation, magnetic incremental permeability, Eddy current testing, Dodd and Deeds model, Jiles-Atherton model. 


\section{1-Introduction:}

Electromagnetic Non-Destructive Testing (ENDT) regroups a large set of methods based on the electromagnetic signature of tested specimens [1]. The objective is to use these signatures as an indirect way to evaluate a level of integrity.

Eddy Current Testing (ECT) is by far the most popular ENDT. Despite its relative simplicity, it conveys a large amount of information. Different configurations of ECT have already been described in the scientific literature:

_ The single coil method [2].

_ The Transmitter/Receiver (TR) probe method [3].

_ The differential probe method [4].

If the specimen is ferromagnetic, the superimposition of a slowly varying high amplitude magnetic excitation to the ECT characterization enables the measurement of the Magnetic Incremental Permeability (MIP) signature (the butterfly loop: incremental permeability plotted as a function of the static magnetic excitation field leading typically to a butterfly shape). MIP is interesting as it is a remarkable indicator of residual stresses and strains [5]-[7]. When ECT is mostly used to detect surface macroscopic flaws (cracks, grinding burns ...), MIP is more adapted to evaluate the mechanical sub-surface properties of tested specimens and their evolutions (creep, structural health monitoring ...).

ENDT is daily used in the steel industry. Due to their high electrical conductivities, industrial steels are well indicated for ECT method. Most of steel alloys are ferromagnetic. From the NDT point of view, this property can be seen as an advantage, it opens access to a large panel of NDT methods exclusive to the ferromagnetic materials (the magnetic Particle inspection (MPI) [8], the Magnetic 
Barkhausen Noise (MBN) [9][10], the Harmonic Analysis (HA) [11] ...). The magnetic behavior of ferromagnetic steels is depending on the micro-structure and on the mechanical internal properties. The magnetic answer of a tested specimen under the influence of an external magnetic stimuli constitutes an indirect way to evaluate these properties. In [12][13] authors have demonstrated the viability of the magnetic methods for the observation of the ductilebrittle transition temperature (DBTT) and the Vickers hardness of structural materials used for nuclear reactor pressure vessels. But ferromagnetism is also strongly nonlinear. When it comes to the interpretation of ENDT signals such nonlinear behavior constitutes an obstacle in the development and the spread of ENDT methods.

The capability of ENDT is well admitted in the industrial field but progresses in this domain are still highly expected. Among many options already tested to improve the efficiency of ENDT, the development of simulation tools seems especially promising. By combining simulation results and experimental data, the ENDT signals can be anticipated and used to prevent possible failures [14]. Different methods have been developed for the simulation of ENDT industrial situations. A space discretization of the Maxwell equations is one solution [15], analytical approaches is another one [16]. In most of the commercial simulation tools dedicated to ENDT, hysteresis is ignored and the relation between the magnetic stimuli and the magnetic state is always taken into account through linear relations. This restriction is clearly an issue, especially for the simulation of the magnetic methods (MBN, MIP ...).

The absence of hysteresis in commercial simulation tools is due to multiple reasons, among these: 
- the complexity of hysteresis and the complexity of hysteresis models. The combined simultaneous resolution of the Maxwell equation and of a hysteresis model is most of the time indirect and needs the use of dedicated techniques such as the fixed point or the NewtonRaphson methods [17][18].

_ the difficulty of gathering reliable magnetic experimental characteristics, as required by the hysteresis model settings.

Progresses have already been done concerning the simultaneous resolution. Strong formulations coupling an hysteresis material law to the Maxwell equations resolution have been proposed and seem to provide positive results [19][20]. In the same time, very few scientific research works have been done to solve the problem of the experimental results acquisition.

In this manuscript, by coupling numerical tools, optimization parameter methods and experimental results accessible from classic NDT equipment, we propose a technique able to return the hysteresis simulation parameters. As a consequence, the geometry has no more influence on the results, and the method is useful even on oversize specimens impossible to characterize with the hysteresis characterization standard devices.

The first step of the method consisted in the acquisition of the MIP curve, evolution of the ECT single coil impedance modulus variations as a function of a DC magnetic field excitation $|Z|(H)$. Standard ECT and MPI equipment can be used to achieve these measurements. D\&D simulations were performed on a large window of simulation parameters (frequency $f$, permeability modulus $|\mu|$ and a reference surface $Z(f,|\mu|)$ was plotted. This surface constituted the support of the interpolation inverse procedure run to return the permeability vs the magnetic excitation curve $|\mu|(H)$. In the last step of the process, the J-A model in a slightly modified configuration was 
operated to simulate the MIP behavior and plot the corresponding $|\mu|(H)$ curves. Again, large windows of parameters were tested, the confrontation between the experimental and the simulated $|\mu|(H)$ curves allowed to establish the best J-A combination of parameters. These parameters were set for the simulation of the MIP curve and conserved for the simulation of the hysteresis behavior $B(H)$.

The structure of this manuscript can be summarized as follows:

The first section describes the experimental setup and the acquisition of the $|Z|(H)$ experimental results. A description of the $D \& D$ simulation method such as the inverse process to obtain the $Z$ $(f,|\mu|)$ is proposed in the second section. The J-A model in its modified version is detailed in the third section and finally the whole process is run and tested on electric steel specimens. Comparisons between simulated hysteresis cycles and experimental ones are proposed as a validation of the hysteresis parameter combination such as the validation of the whole method.

\section{2 - Experimental setup, acquisition of the $|Z|(H)$ experimental results}

\section{2-a) Magnetic incremental permeability characterization setup}

The first step of this research consists in the acquisition of the MIP butterfly signature: evolution of the sensor coil impedance modulus $|Z|$ as a function of the tangent magnetic field excitation $H$. Standard NDT industrial equipment can be used for this acquisition (Fig. 1). The DC magnetic excitation can be generated through a MPI experimental setup and the evolution of the flat coil impedance monitored with an eddy current controller (Fig. 1). 


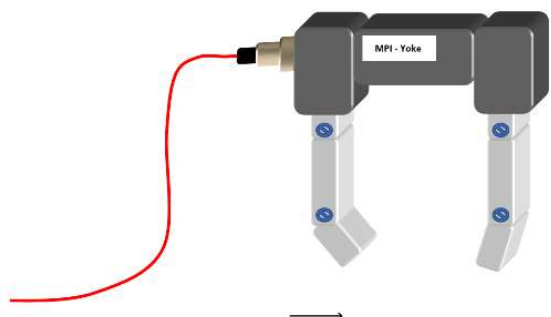

$\mathrm{MPI}$, high amplitude $\overrightarrow{H_{D C}}$ magnetization
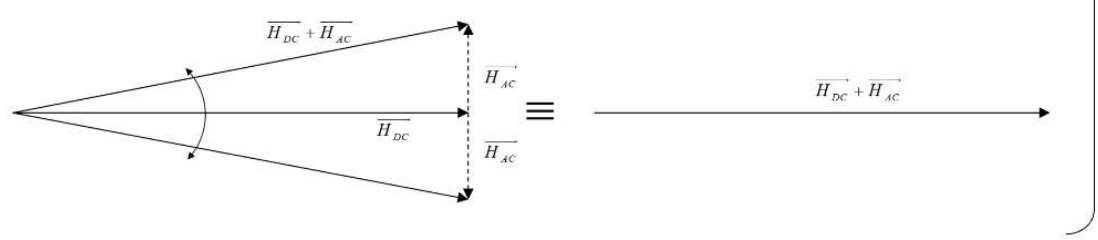

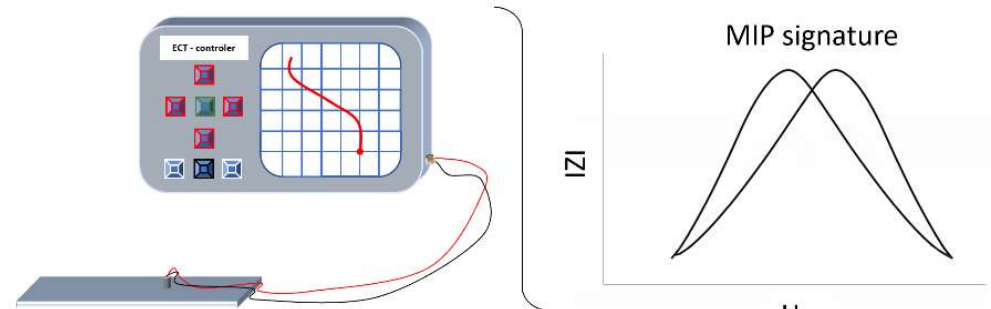

$\mathrm{H}$

ECT, $\overrightarrow{H_{A C}}$ sensor coil impedance
ECMS signature

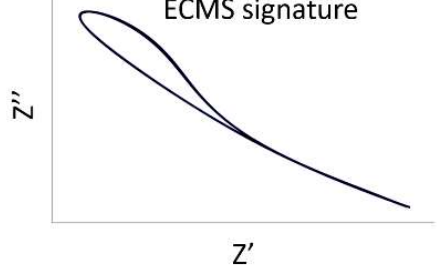

Fig. 1 - Illustration of the MIP and ECMS signature acquisitions with standard NDT equipment.

In our laboratory, our equipment is slightly different. A dedicated experimental setup has been developed especially to recreate the industrial conditions. An overall 3D view of this setup is depicted in Fig. 2 below.

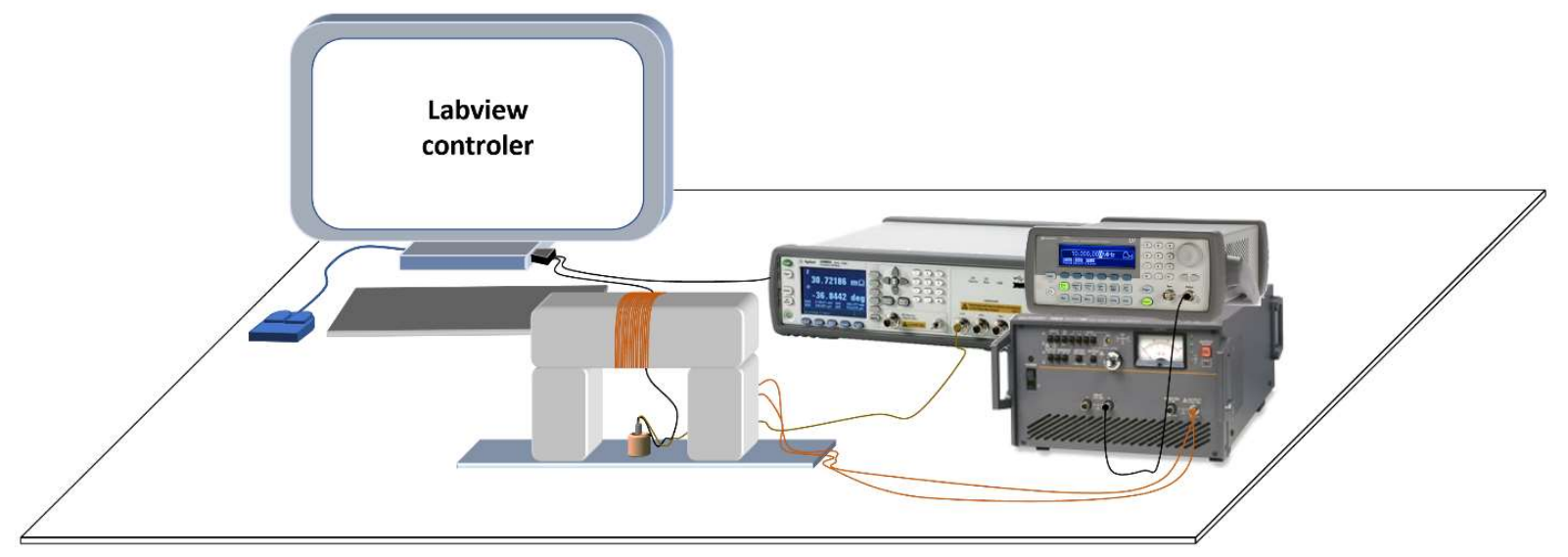

Fig. 2 - Overall 3D view of the industrial setup used for the acquisition of the incremental permeability.

A DC power amplifier (HSA 4014 by NF Corporation) fed in $f=0.01 \mathrm{~Hz}$ current a 200 turns wound coil. (please note that to get closer to the industrial conditions, this amplifier and the associated frequency generator can be replaced by a regulated power supply controlled manually. If $f$ is low 
enough both methods give the same MIP curve. We did this test to set $f$ and avoid any undesired dynamic contribution). The exciting coil is associated to a U-shaped large section magnetic yoke for the generation of the $\mathrm{H}_{D C}$ magnetic field excitation contribution. The tangential magnetic field along the specimen in the field direction was measured using a Hall element. A pancake coil (ECT coil) was placed on the surface of the specimen between the legs of the yoke (see Fig. 1). The dimensions of the ECT coil were as follows: $3.3 \mathrm{~mm}$ inner diameter, $3.95 \mathrm{~mm}$ outer diameter, 3.0 $\mathrm{mm}$ height, 275 turns, and $0.05 \mathrm{~mm}$ wire thickness. The lift-off was $0.39 \mathrm{~mm}$. The coil impedance was measured using a LCR meter (ZM2375, NF Corporation). The current of the ECT coil was controlled to an effective value of $1 \mathrm{~mA}$ using the LCR meter. The frequency of this AC magnetic field excitation was set to $50 \mathrm{KHz}$. The $\mathrm{AC}$ magnetic field contribution was maintained lower than a quarter of the coercivity to ensure reversible $A C$ magnetization changes, i.e., to avoid irreversible domain wall jumps, as recommended by the literature [21]. The resulting data provided by the LCR meter consisted in the sensor coil impedance modulus, phase, real and imaginary part. A series of measurements for the different levels of DC magnetization was recorded using @Labview data acquisition software.

A calibration procedure was carried out to take into account the parasitic impedances due to the electrical wires and contacts. A comparison between experimental and simulation tests in the air (using the D\&D analytical expression described in the next section for the simulation) was done and both a rotation $\varphi_{c o r}$ and expansion $\operatorname{Mod}_{c o r}$ factors were found so that the calculation results were consistent with the experimental ones.

$$
Z_{f i n}=|Z| \cdot \operatorname{Mod}_{c o r} \cdot e^{j\left(\varphi+\varphi_{c o r}\right)}
$$


This method gave us the MIP signature but it also enabled access to the Eddy Current Magnetic Signature (EC-MS) plot, impedance imaginary part as a function of the real part which has been described in recent articles as a particularly sensitive indicator of residual strains [6][22].

In parallel to this first experimental setup, a hysteresis cycle characterization setup was exploited to plot the tested specimen hysteresis cycles required for the validation of the method. A detailed description of this setup can be found in [22].

Fig. $3-a, b$ give the MIP and the EC-MS curves obtained at $H_{A C}=50 \mathrm{Khz}$. Fig. $3-\mathrm{c}$ shows the experimental quasi-static major hysteresis cycle measured with our specimens.
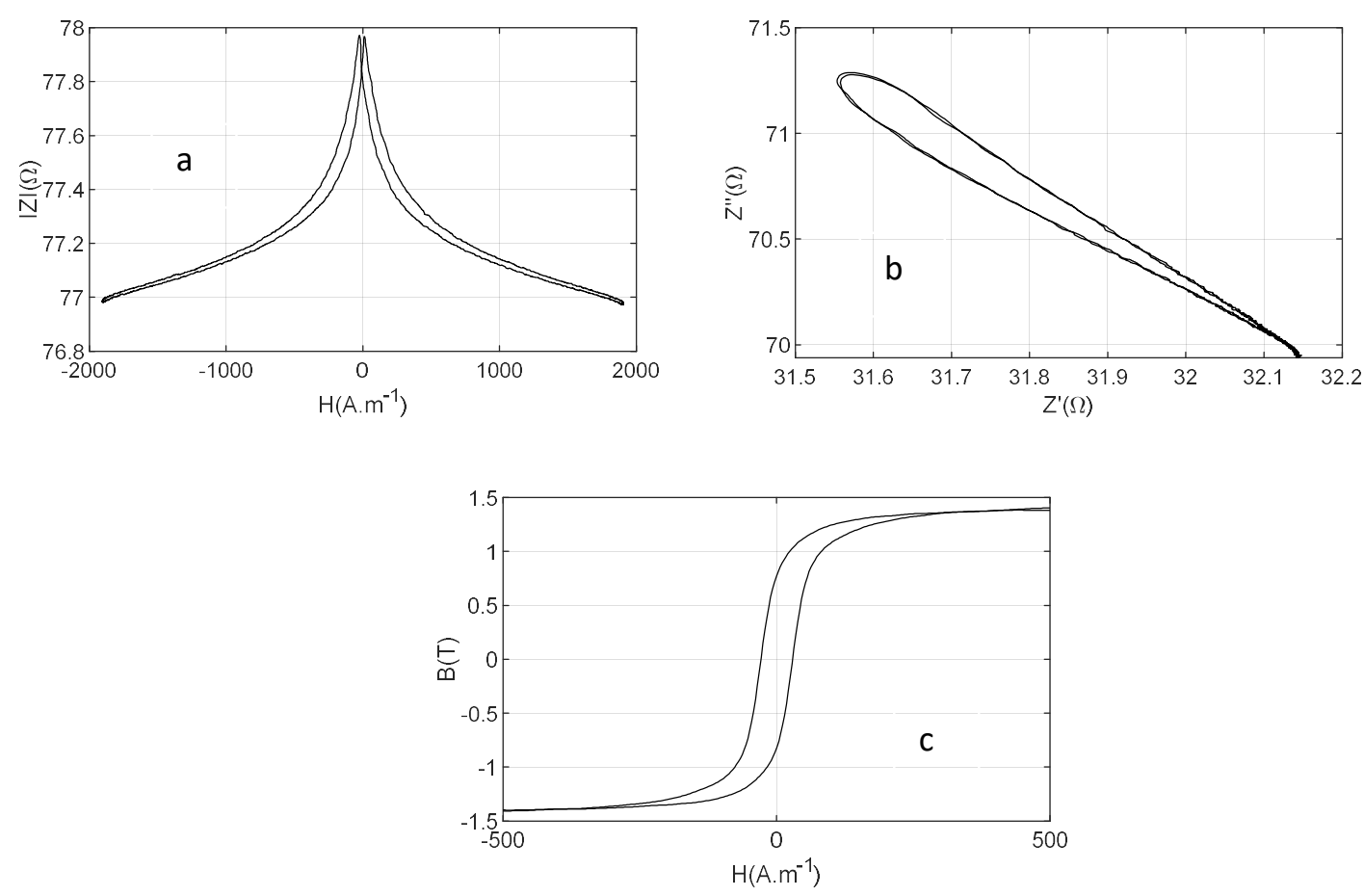

Fig. 3 - a. MIP Experimental curve $\left(H_{A C}=50 \mathrm{KHz}\right)$. Fig. 3 - b. ECMS Experimental curve $\left(H_{A C}=50 \mathrm{KHz}\right)$. Fig. $3-$ c. $B(H)$ Experimental curve $\left(H_{D C}=0.1 \mathrm{~Hz}\right)$. 


\section{2-b) Tested specimens}

The tested specimens were all coming from the same batch. They were all electric steel, FeSi laminations with a $3 \mathrm{wt} \%$ silicon content and referenced 35JN360. Tab. 1 below gives the physical properties as provided by the manufacturer.

Tab. 1 - Physical properties of the 35JN360 specimens as provided by the manufacturer.

\begin{tabular}{|c|c|c|c|c|c|c|c|c|}
\hline \multirow[b]{2}{*}{ Grade } & \multirow[t]{2}{*}{$\begin{array}{l}\text { Thickness } \\
\text { (mm) }\end{array}$} & \multirow[t]{2}{*}{$\begin{array}{l}\text { Density } \\
\left(\mathrm{kg} / \mathrm{dm}^{3}\right)\end{array}$} & \multicolumn{2}{|c|}{$\begin{array}{l}\text { Core loss - } 50 \mathrm{~Hz} \\
\quad(\mathrm{~W} / \mathrm{kg})\end{array}$} & \multicolumn{3}{|c|}{$\begin{array}{l}\text { Min. magnetic flux density at } 5000 \text { A.m-1 } \\
\text { (T) }\end{array}$} & \multirow[t]{2}{*}{$\begin{array}{l}\text { Min. Lamination Factor } \\
\text { (\%) }\end{array}$} \\
\hline & & & $1 \mathrm{~T}$ & $1.5 \mathrm{~T}$ & & & & \\
\hline \multirow[t]{3}{*}{$35 \mathrm{JN} 360$} & 0.35 & 7.65 & 1.45 & 3.6 & & & & 95 \\
\hline & \multicolumn{2}{|c|}{$\begin{array}{l}\text { Yield point } \\
\left(\mathrm{N} / \mathrm{mm}^{2}\right)\end{array}$} & \multicolumn{2}{|c|}{$\begin{array}{l}\text { Tensile Strength } \\
\mathrm{N} / \mathrm{mm} 2\end{array}$} & \multicolumn{2}{|c|}{$\begin{array}{c}\text { Elongation } \\
\%\end{array}$} & $\begin{array}{l}\text { Hardness } \\
\text { HV (1 kg) }\end{array}$ & \\
\hline & $\begin{array}{c}\mathrm{L} \\
351\end{array}$ & $\begin{array}{c}C \\
356\end{array}$ & $\begin{array}{c}\mathrm{L} \\
489\end{array}$ & $\begin{array}{c}C \\
04\end{array}$ & $\begin{array}{c}\mathrm{L} \\
27\end{array}$ & $\begin{array}{c}\mathrm{C} \\
28\end{array}$ & 182 & \\
\hline
\end{tabular}

"L" and " $\mathrm{C}$ " means the specimens cut parallel and traverse to the rolling direction, respectively.

The tested specimen geometrical information are available in Fig. 4.

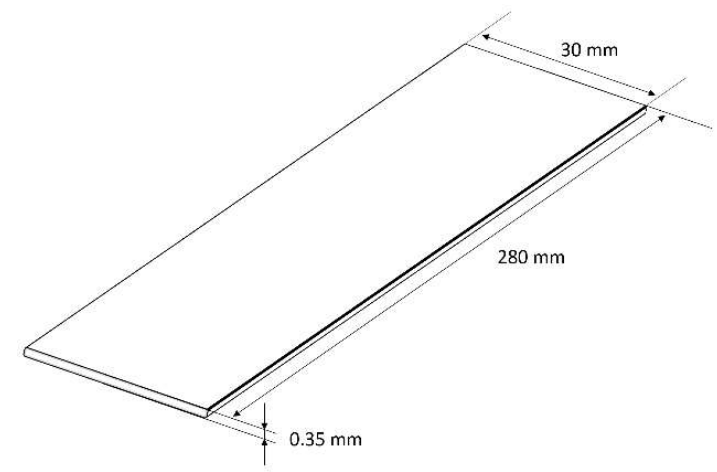

Fig. 4-Geometrical properties of the tested specimens.

The electrical conductivity of these specimen was $2.1310^{6} \mathrm{~S} \cdot \mathrm{m}^{-1}$, with Non-Oriented (NO) grains and isotropic magnetic properties. 


\section{3 - Inverse process, Dodd and Deeds analytical method, $|\mu|(H)$ plots establishment.}

In the late sixties, early seventies, Dodd and Deeds (D\&D) derived closed form integral expressions for the impedance of the cylindrical coil above a layered conductive half-space [23][25]. This model continues to be used extensively to this day. In the industrial field, it allows to design eddy current tests and to optimize parameter measurements including thickness, conductivity and magnetic permeability [26]-[28]. In this study, the D\&D simulation method was used to plot a reference surface $|Z|\left(\left|\mu_{r}\right|, f\right)$ related to our experimental situation. This surface constituted the base of the interpolation method used to convert the experiment $|Z|(H)$ plots into $\left|\mu_{r}\right|(H)$ plots.

We opted for the D\&D analytical solution of a cylindrical coil above a two-layer conductor. The top layer was our tested specimen ( $0.35 \mathrm{~mm}$ thick), the bottom layer (supposed extended to infinity) was set with a vacuum permeability and a low electrical conductivity in order to restrain its influence in the final calculus. The D\&D solution is decomposed in two terms. The first term $Z_{0}$ is related to the cylindrical coil and is expressed by:

$$
Z_{0}=\frac{j 2 \pi \omega \mu_{0} N^{2}}{\left(r_{2}-r_{1}\right)^{2}\left(z_{2}-z_{1}\right)^{2}} \int_{0}^{+\infty} \frac{\chi^{2}\left(k r_{1}, k r_{2}\right)}{k^{6}}\left[k\left(z_{2}-z_{1}\right)+e^{-k\left(z_{2}-z_{1}\right)}-1\right] d k
$$

Where $r_{1}, r_{2}, z_{1}, z_{2}$, are the coil inner radius, the outer radius, the bottom position and the top position on the $\mathrm{z}$ axis (see Fig. 5 below). $\mathrm{N}$ is the number of turns. 


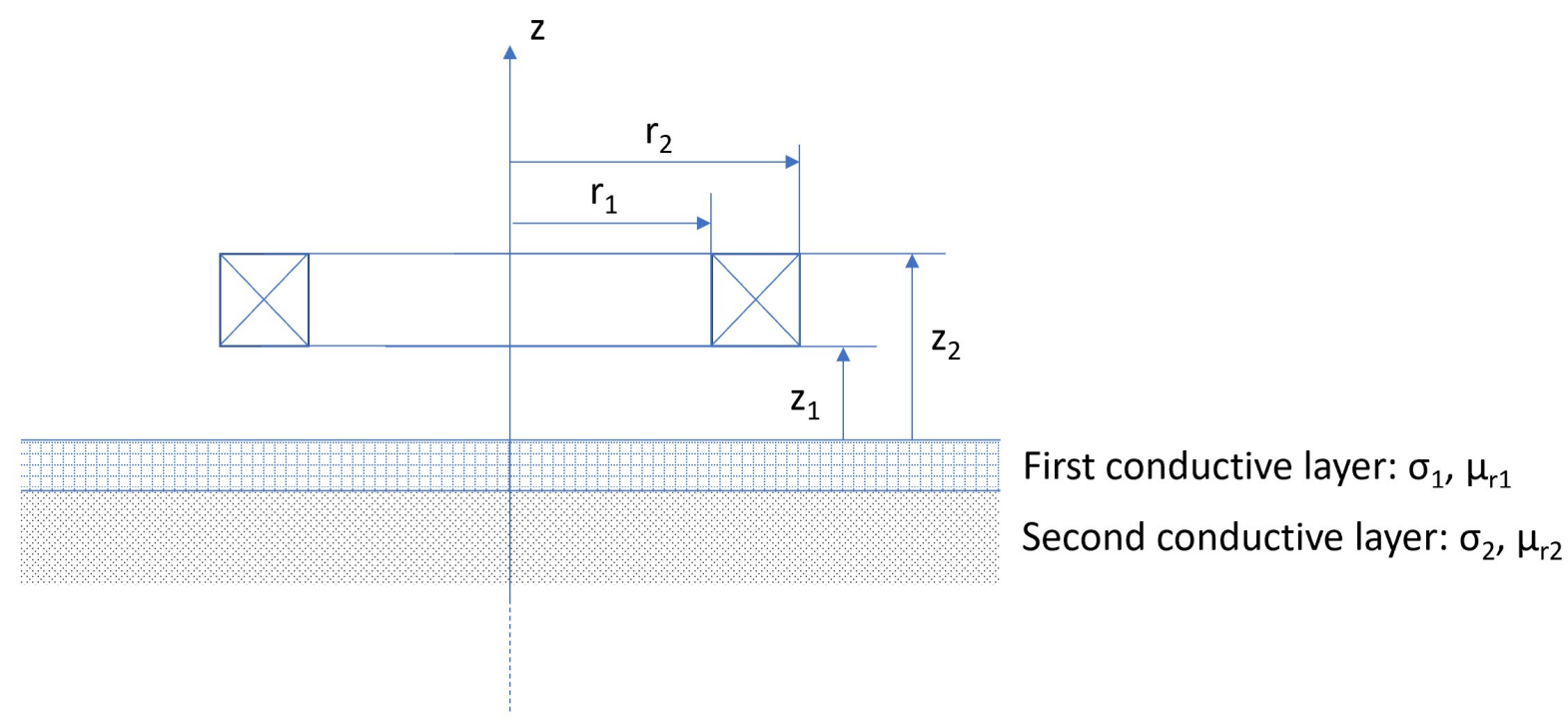

Fig. 5 - Illustration for the D\&D simulation of cylindrical coil above a layered conductive half-space.

$Z_{0}$ does not depend on the vertical position of the coil but rather on its width $z_{2}-z_{1}$. The second term $\Delta Z$ has to be added to $Z_{0}$ to obtain the final solution. $\Delta Z$ is associated to the impedance changes due to the two-layers conductor and can be expressed as:

$\Delta Z=\frac{j 2 \pi \omega \mu_{0} N^{2}}{\left(r_{2}-r_{1}\right)^{2}\left(z_{2}-z_{1}\right)^{2}} \int_{0}^{+\infty} \frac{\chi^{2}\left(k r_{1}, k r_{2}\right)}{k^{6}}\left(e^{-k z_{1}}-e^{-k z_{2}}\right)^{2} \frac{\left(\lambda_{1} \mu_{r 2}+\lambda_{2} \mu_{r 1}\right)\left(k \mu_{r 1}-\lambda_{1}\right)+e^{-2 \lambda_{1} d}\left(\lambda_{1} \mu_{r 2}-\lambda_{2} \mu_{r 1}\right)\left(k \mu_{r 1}+\lambda_{1}\right)}{\left(\lambda_{1} \mu_{r 2}+\lambda_{2} \mu_{r 1}\right)\left(k \mu_{r 1}+\lambda_{1}\right)+e^{-2 \lambda_{1} d}\left(\lambda_{1} \mu_{r 2}-\lambda_{2} \mu_{r 1}\right)\left(k \mu_{r 1}-\lambda_{1}\right)} d k$

$$
\lambda_{i}=\sqrt{k^{2}+j \omega \mu_{r_{i}} \mu_{0} \sigma_{i}}
$$

Where $d$ is the first conductive layer thickness. The D\&D analytical solution was configured with the sensor coil information provided in the first section and simulations were done for a frequency and relative permeability windows of $[10 \mathrm{KHz}-1 \mathrm{MHz}]$ and [100 - 5000] respectively. The resulting surface $|Z|\left(\left|\mu_{r}\right|, f\right)$ is depicted in Fig. 6. 


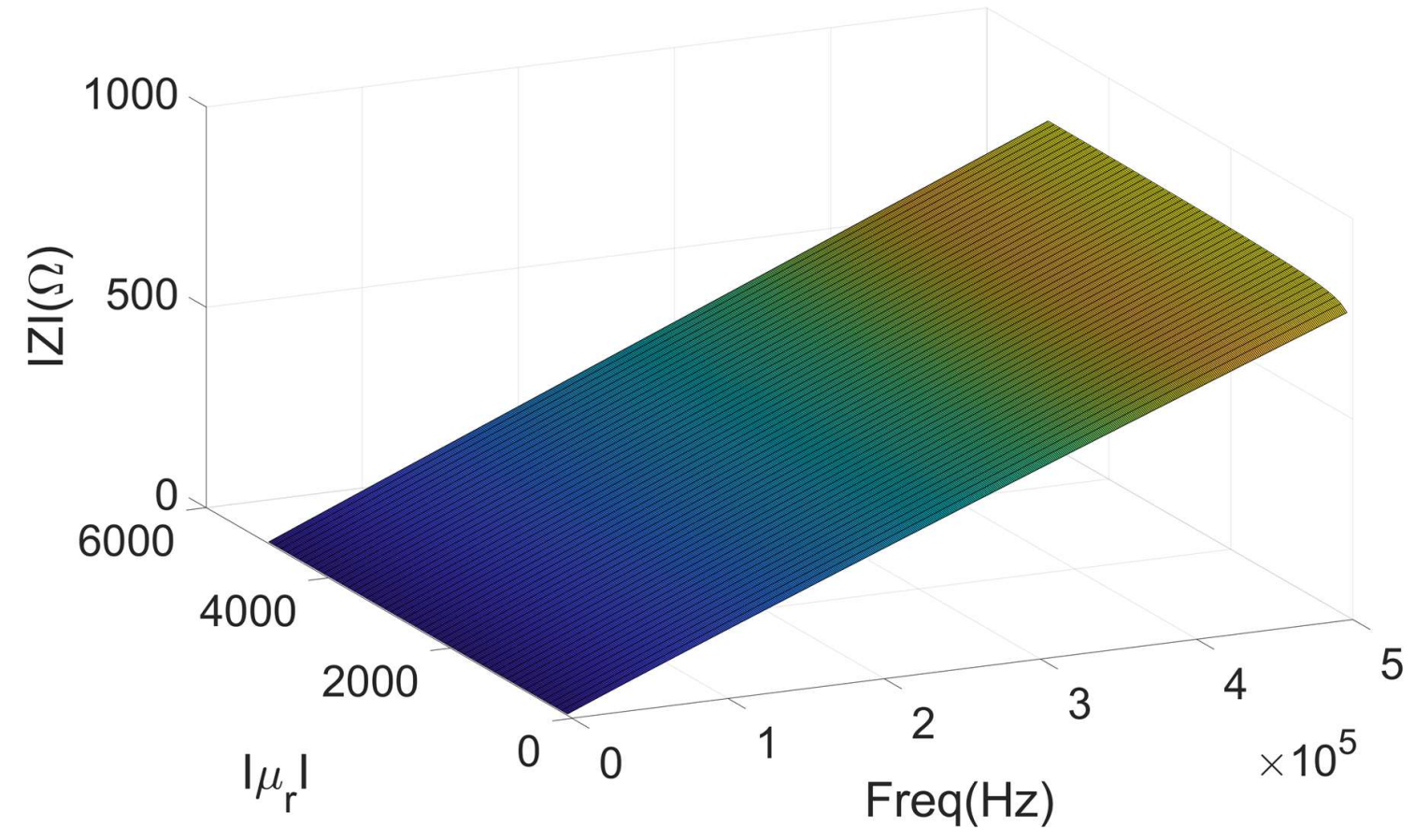

Fig. 6 - Reference surface $|Z|\left(\left|\mu_{r}\right|, f\right)$ calculated with the D\&D simulation method.

Fig. 6 surface and the corresponding matrix constitutes the data base we use to return by interpolation the relative permeability corresponding to Fig. 3 IZI experimental values. Even if the experimental AC frequency was set to $50 \mathrm{Khz}$, a large window of frequencies was tested making our method applicable on different levels of frequencies.

Fig. 7 - a below depicts the simulated $\left|\mu_{r}\right|(|Z|)$ curve obtained at $f=50 \mathrm{KHz}$, Fig. $7-\mathrm{b}$ shows the $\left|\mu_{r}\right|(H)$ curve obtained for our specimen once the correction factors were taken into account and the interpolation process done. The expansion factor $\operatorname{Mod}_{c o r}$ was set to $\times 1.1896$ and the rotation factor $\varphi_{\text {cor }}$ to $-0.4511 \mathrm{rd}$. 

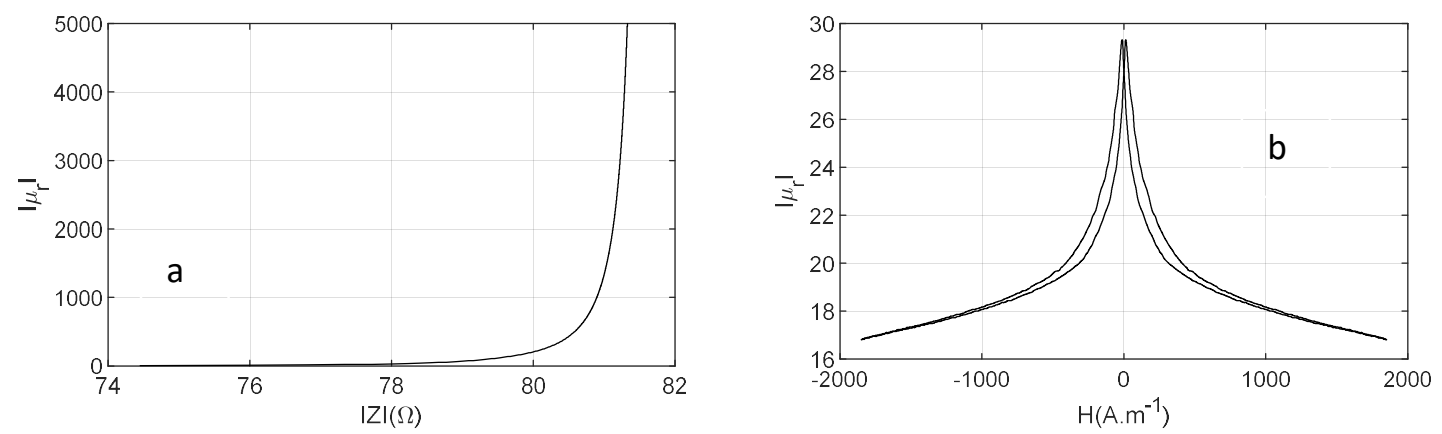

Fig. 7 - a. Relative permeability as a function of the impedance from the D\&D simulation $(f=50 \mathrm{KHz})$. Fig. $7-\mathrm{b}$. $\left|\mu_{r}\right|(H)$ resulting curve for the FeSi 3\% NO specimen.

\section{4 - Modified Jiles-Atherton model and identification of the hysteresis parameters.}

The inverse process based on the D\&D analytical solution for a cylindrical coil above a layered conductive half-space gave us an estimation of $\left|\mu_{r}\right|$ for all the values of $\mathrm{H}$ tested. It is important to keep in mind that $\mu_{\mathrm{r}}$ is a tensor linking vector quantities. As the tested specimen is magnetized by the $\mathrm{DC}$ magnetic field contribution, $\left|\mu_{r}\right|$ is supposed to be independent of the spatial position. Even if we never verified it experimentally, our specimens are supposed to have anisotropic magnetic characteristics (non-oriented grains). In our experimental setup, the $A C$ and the DC magnetic contributions were perpendicular (see Fig. 1). The quasi-static excitation amplitude is supposed to be 100 to 1000 times higher than the $A C$ ones. Consequently, in the scanned area the direction of the vector cumulative excitation field $\vec{H}\left(\overrightarrow{H_{D C}}+\overrightarrow{H_{A C}}\right)$ remains almost constant during a large proportion of the measurement cycle. Based on this observation, we considered the scalar version of the Jiles-Atherton model (where $\vec{H}$ and $\vec{B}$ are collinear) as accurate enough to provide consistent simulation results. 


\section{4-a) Modified Jiles-Atherton model for a fast simulation of the magnetic incremental permeability}

The J-A theory is based on domain-wall motion which is the principal cause of hysteresis in multidomain specimens. But the J-A model also considers the effect of domain rotation since the essence of the model is the establishment of a relationship between energy dissipation ("Iosses") and magnetization variations [29]-[31]. On a one hand, the J-A model exhibits remarkable properties: a limited number of parameters, a capability of considering hysteresis through a unique equation. On the other hand, the J-A model (at least, in its early stage version) is suffering from various restrictions: the limitation to scalar situation (i.e., the magnetic excitation $\mathrm{H}$ and the magnetization $M$ are assumed to be collinear), the limitation to quasi-static frequencies and the accommodation issue (i.e., the incapability to simulate correctly the minor loop situations). In the J-A theory, $M$ is decomposed into a reversible $M_{\text {rev }}$ and an irreversible $M_{\text {irr }}$ contributions:

$$
M=M_{r e v}+M_{i r r}
$$

The anhysteretic magnetization is described with a sigmoid-type equation (Langevin ...) including at least two parameters:

_ $M_{s}$ the saturation magnetization

_ $a$, an anhysteretic trajectory parameter related to the domain walls density.

Eq. 5 and eq. 6 below are the classic options for this anhysteretic magnetization:

$$
\begin{aligned}
& M_{a n h}=M_{S}\left[\operatorname{coth}\left(\frac{H_{e}}{a}\right)-\frac{a}{H_{e}}\right] \\
& M_{a n h}=M_{s} \tanh \left(\frac{H_{e}}{a}\right)
\end{aligned}
$$

According to the J-A theory, a can obtained theoretically using Eq. 7:

$$
a=\frac{k_{b} \theta}{\mu_{0} m}
$$


$k_{B}$ is the Boltzmann's constant, $\theta$ the temperature, and $m$ the magnetic magnitude at a domain scale. In the J-A model, an effective field $H_{e}$ is defined as the sum of $H$ and an additional contribution related to the surrounding magnetized mater and modulated by $\alpha$ a mean field parameter (the inter domain coupling parameter, see J-A theory [29]-[31]).

$$
H_{e}=H+\alpha M
$$

$H_{e}$ is the model input, by taking into account the influence of the environment magnetic state, congruency issues are avoided. Eq. 9 below connects the anhysteretic, the reversible and the irreversible magnetizations:

$$
M_{\text {rev }}=c\left(M_{\text {anh }}-M_{\text {irr }}\right)
$$

As $\mathrm{c}$ is constant, a proportional ratio is assumed between the magnetization contributions. c can be adjusted experimentally from the ratio between the initial permeability observed on the first and on the anhysteretic magnetization curves (in the Rayleigh area). c has a strong influence in the simulation of the MIP butterfly signature where similarly to the Rayleigh zone, the reversible magnetization contribution is major.

A differential equation (Eq. 10) connects the anhysteretic and the irreversible magnetization:

$$
\frac{d M_{i r r}}{d H_{e}}=\frac{M_{a n}-M_{i r r}}{k \delta}
$$

$k$ is related to an average energy required by the domain walls to break pinning site and $\delta$ a directional parameter necessary to avoid physical non-sense.

$$
\left\{\begin{array}{l}
\delta=+1 \text { if } \quad \frac{d H}{d t} \geq 0 \\
\delta=-1 \text { if } \frac{d H}{d t}<0
\end{array}\right.
$$

After recombination of the above equation (Eq.4 to 11) an expression of the susceptibility (assumed equal to the permeability) can be obtained: 


$$
\frac{d M}{d H}=\frac{(1-c) \frac{d M_{i r r}}{d H_{e}}+c \frac{d M_{a n h}}{d H_{e}}}{1-\alpha(1-c) \frac{d M_{i r r}}{d H_{e}}-\alpha c \frac{d M_{a n h}}{d H_{e}}}
$$

Eq. 12 permeability can be classified as differential if $d H / d t$ has been conserving the same sign for a time lap long enough to allowed irreversible domain wall motions. Oppositely, after a recent $d H / d t$ sign changing, it can be assimilated to an incremental permeability. The $H$ integration of eq. 12 leads to the induction field $B$ and allow to plot the $B(H)$ hysteresis behavior:

$$
B(t)=\mu_{0}\left(H(t)+\int \frac{d M}{d H}(t) d H\right.
$$

In [22], we proposed a simple modification of the J-A model to describe quasi simultaneously a $B(H)$ hysteresis cycle and the associated $\left|\mu_{r}\right|(H)$ MIP butterfly loop. In this method, the J-A model is excited with a high amplitude alternative magnetic field $\mathrm{H}$ and for every step time both the differential and the incremental magnetic permeability are calculated. In such configuration, $H$ variations are long enough to allow irreversible domain wall motions and eq. 12 describes a differential permeability. The incremental permeability calculus requires the creation of a virtual MIP effective field $\mathrm{He}_{\text {MIP }}$ defined as:

$$
\left\{\begin{array}{c}
\text { if sign }\left(\frac{d H(t)}{d t}\right)=\operatorname{sign}\left(\frac{d H(t-d t)}{d t}\right) \\
H_{e_{M I P}}(t)=H(t-d t)-d H(t)+\alpha M(t-d t) \\
\text { else } \\
H_{e_{M I P}}(t)=H_{e}(t)
\end{array}\right.
$$

$H e_{\text {MIP }}$ simulates a sudden sign change in the excitation field time derivation and replacing He by $H e_{\text {MIP }}$ in the simulation process leads to a permeability expression (Eq. 15) which can be assimilated to the incremental permeability:

$$
\frac{d M}{d H}_{M I P}=\frac{(1-c) \frac{d M_{i r r}}{d H_{M I P}}+c \frac{d M_{a n h}}{d H_{e_{M I P}}}}{1-\alpha(1-c) \frac{d M_{i r r}}{d H_{e_{M I P}}}-\alpha c \frac{d M_{a n}}{d H_{e_{M I P}}}}
$$


Fig. 8 below gives an example of a simulated major $B(H)$ hysteresis cycle and its related differential and incremental permeability obtained with the modified J-A model and the combination of parameters displayed in Fig. $8-$ b.
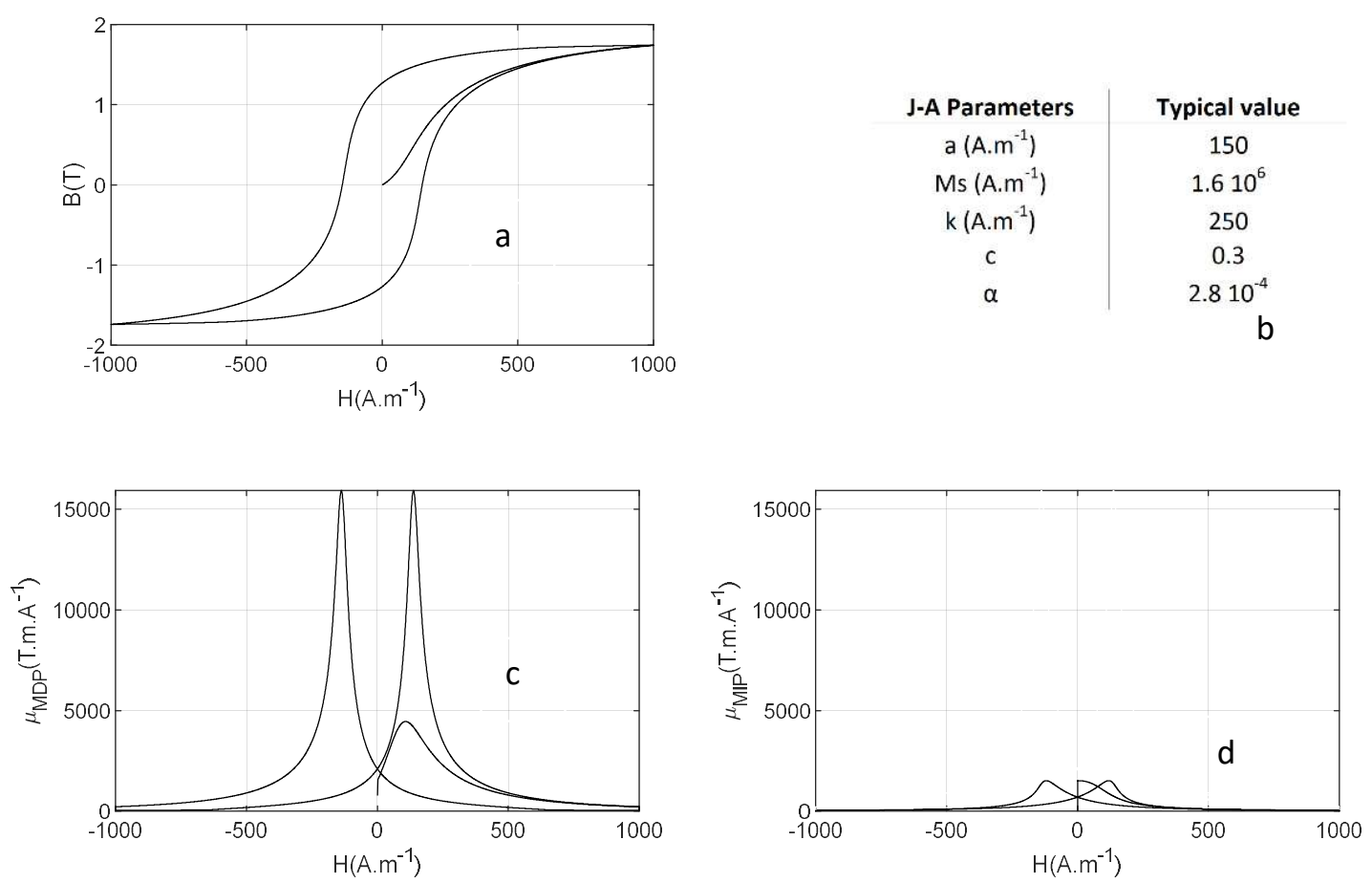

Fig. 8 - a. Simulated B(H) major hysteresis cycle. Fig. 8 - b. Corresponding J-A parameters. Fig. 8 - c. Simulated MDP curve. Fig. 8 - d. Simulated MIP curve.

By avoiding the simulation of the minor loops, the new method solves indirectly the accommodation issue and provides faster simulation results. According to numerical tests commented in [22], the quantitative improvement reaches $x 10$ in term of simulation time which is large enough to envisage setting up a parameter optimization process and find the best combination of parameter for the simulation of Fig.6 $\left|\mu_{r}\right|(H)$ curve.

The optimization process constituted the next step of this work. The J-A parameters were identified precisely, the optimization process was based on comparisons between Fig. $7-b$ $\left|\mu_{r}\right|(H)$ D\&D interpolated curves and the J-A simulated ones. Windows of values based on 
physical properties were set for every parameters. Ms was adjusted from the material composition.

$$
\begin{gathered}
\frac{d H}{d t}>0, H_{i} \in\left[H_{\text {min }}, H_{\text {max }}\right] \\
\text { Error }_{\text {MIP }}=\sum_{i=1}^{n} \operatorname{abs}\left(|\mu|_{i}^{D \& D}\left(H_{i}\right)-|\mu|_{i}^{J-A}\left(H_{i}\right)\right)
\end{gathered}
$$

The combination of parameters which lead to the minimum value of the error function (Eq. 16) was set as our specimen combination of parameters. This combination is depicted in Tab. 2. Eq. 5 sigmoid has been used for the J-A anhysteretic contribution.

Tab. 2 - Best combination of parameters for the J-A simulation of the 35JN360 specimens.

$\begin{array}{cc}\text { J-A Parameters } & \text { 35JN360 specimens } \\ \left.\text { a (A. } \mathrm{m}^{-1}\right) & 16000 \\ \text { Ms }\left(\text { A. } \text { m }^{-1}\right) & 7910^{5} \\ \left.\text { k (A. } \mathrm{m}^{-1}\right) & 25 \\ \mathrm{C} & 0.0015 \\ \alpha & 0.0001\end{array}$

Finally, both the D\&D and the J-A (Tab. 2 parameters) $\left|\mu_{r}\right|(H)$ curves are exposed in Fig. $9-$ a. Fig. 9 - b shows the resulting comparison between the experimental and the simulated $B(H)$ curves.
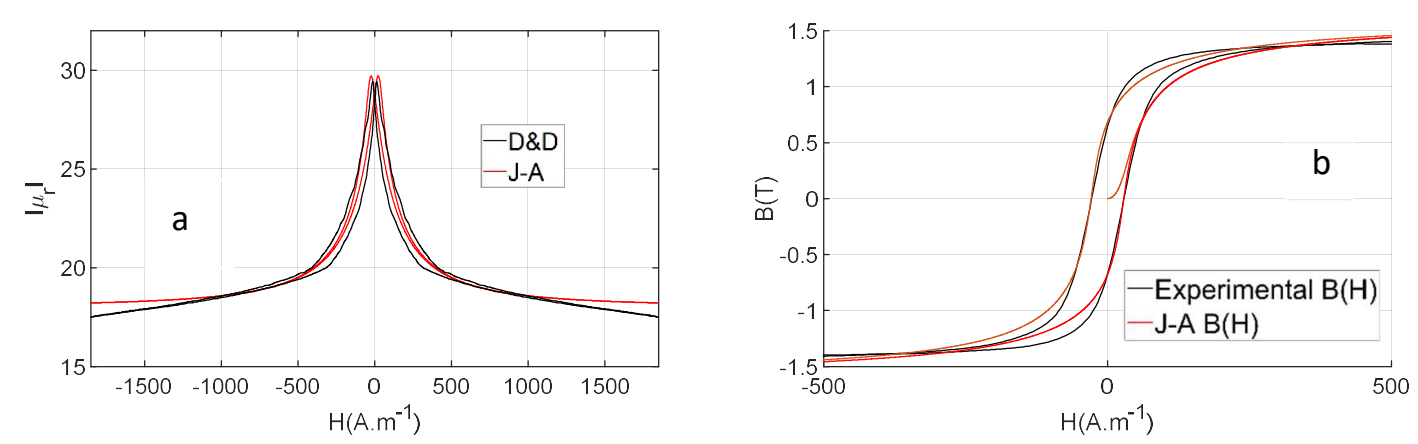

Fig. 9 - a. Comparison between the IZI(H) MIP curves obtained with the D\&D interpolation method and with the JA model (best combination of parameters). Fig. $9-$ b. Comparison between the simulated and the measured $B(H)$ curves 
The relatively good comparison observed between the experimental and the simulated $B(H)$ curves (Fig. $9-$ b) confirms our expectation and is worth as a validation of the whole method. A short quantitative survey (relative error percentage (Eq. 16) on the coercive field, the remnant induction and the hysteresis area) is depicted in Tab. 3 below.

$$
\operatorname{Error}_{\text {rel }}(\%)=100\left|\frac{X_{\text {meas }}-X_{\text {sim }}}{X_{\text {meas }}}\right|
$$

Tab. 3 -Relative error percentage for 3 hysteresis cycle parameters.

\begin{tabular}{c|c} 
& Rel. Error (\%) \\
\hline Ec & 3.2 \\
Br & 6 \\
Hyst area $<$ A $>$ & 21
\end{tabular}

Finally, it is important to mention the weight of the lift off estimation in the success of the method. Large differences can be observed on the $\left|\mu_{r}\right|(|Z|)$ (Fig. $\left.7-a\right)$ and on the $\left|\mu_{r}\right|(H)$ (Fig. 7 -b) curves after a small variation of the lift-off. Good simulation results can still be obtained by adjusting $\mathrm{c}$ (the reversibility parameter). $\mathrm{c}$ has a strong influence on the MIP simulation but relatively weak on the $\mathrm{B}(\mathrm{H})$ curve, it can be modified to match the MIP curve without affecting the $B(H)$ simulation. Consequently, from a magnetic characterization/NDT point of view, a small error in the measurement of the lift-off can lead to a resulting $c$ out of physical meaning.

\section{5 - Conclusions and perspectives}

ENDT is in constant progress, by coupling numerical tools to experimental results, the capability of detection and characterization could be improved drastically. A lot of research is supported in this field. An important current issue comes from the acquisition of the experimental data required for the configuration of the simulation methods. It is especially true for steel inspection. Steel is ferromagnetic and exhibits strong magnetic non-linear behaviors (hysteresis, frequency 
dependence ...). The magnetic characterization of steel is supposed to be done in very restrictive conditions described by international standards. In this manuscript, we proposed an alternative method to estimate the hysteresis simulation parameters using classic NDT equipment (ECT device and MPI inductor). The proposed method runs through 3 steps and combines experimental results (MIP butterfly loop) to numerical simulations (the D\&D analytical solution and the J-A model). The first tests exposed in this manuscript for the FeSi laminations with a 3 wt $\%$ silicon content exhibit a maximum of $\approx 20 \%$ relative error for the main hysteresis indicators. Even if this percentage error can be judged as relatively high, it has to be balanced by the fact that the resulting hysteresis parameters open doors to comparisons between data from NDT experimental devices and situations never compared before.

Many perspectives can be associated to this work including:

_ To test new geometries (tubes, rods, gears ...)

_ To test new materials (steel, pure iron ...)

Assumptions have been advanced in the description of our method, therefore further developments will be proposed in a future work to confirm these hypothesis and improve the capability of the method. A study of reproducibility has to be performed such as the evolution of the simulation parameters as a function of creep and aging. 


\section{References}

[1] S. Huang, S. Wang, "New Technologies in Electromagnetic Nondestructive Testing", Springer, Singapore, 2016.

[2] S. Borovik, Y. Sekisov, "Single-coil eddy current sensors and their application for monitoring the dangerous states of gas-turbine engines", Sensors, 20(7), 2017.

[3] F. Kojima, T. Takagi, T. Matsui, "Inverse methodology for eddy current testing using transmitterreceiver coil probes", AIP Conf. Proc. 700, 643, 2004.

[4] T. Chady, M. Enokizono, R. Sikora, "Crack detection and recognition using an eddy current differential probe", IEEE Trans. Mag., Vol. 35, Iss. 3, pp. 1849-1852, 1999.

[5] B. Gupta, T. Uchimoto, B. Ducharne, G. Sebald, T. Miyazaki, T. Takagi, "Magnetic incremental permeability non-destructive evaluation of $12 \mathrm{Cr}-\mathrm{Mo}-\mathrm{W}-\mathrm{V}$ Steel creep test samples with varied ageing levels and thermal treatments", NDT \& E Int., Vol. 104, pp. 42-50, 2019.

[6] T. Matsumoto, T. Uchimoto, T. Takagi, G. Dobmann, B. Ducharne, S. Oozono, H. Yuya, "Investigation of Electromagnetic Nondestructive Evaluation of Residual Strain in Low Carbon Steels Using the Eddy Current Magnetic Signature (EC-MS) Method", J. of Mag. and Mag. Mat., Vol. 479, pp. 212-221, 2019.

[7] B. Gupta, B. Ducharne, T. Uchimoto, G. Sebald, T. Miyazaki, T. Takagi, “Comparison of electromagnetic inspection methods for creep-degraded high chromium ferritic steels", NDT\&E Int., Vol. 118, 102399, 2021.

[8] Z.D. Wang, Y. Gu, Y.S. Wang, "A review of three magnetic NDT technologies", J. Magn.. Mater., vol. 324, iss. 4, pp. 382-388, 2020.

[9] B. Ducharne, "Micromagnetic nondestructive testing Barkhausen noise vs other techniques", Elsevier book: Barkhausen noise for nondestructive testing and materials characterization in low-carbon steels, pp. 223-238, 2020.

[10] B. Gupta, B. Ducharne, T. Uchimoto, G. Sebald, T. Miyazaki, T. Takagi, "Non-destructive testing on creep degraded $12 \% \mathrm{Cr}-\mathrm{Mo}-\mathrm{W}-\mathrm{V}$ Ferritic test samples using Barkhausen noise", J. of Mag. and Mag. Mat, Vol. 498, 166102, 2019.

[11] G. Dobmann, H. Pitsch, "Magnetic tangential field strength inspection a further ndt-tool for 3MA", in: P. Höller, V. Hauk, G. Dobmann, C.O. Ruud, R.E. Green (eds) Nondestructive Characterization of Materials, Springer, Berlin, 1989.

[12] S. Takahashi, S. Kobayashi, H. Kikuchi, Y. Kamada, "Relationship between mechanical and magnetic properties in cold rolled low carbon steel”, J. Appl. Phys., 100, 113908, 2006.

[13] I. Tomáš, G.Vértesy, S. Pirfo Barroso, S. Kobayashi, "Comparison of four NDT methods for indication of reactor steel degradation by high fluences of neutron irradiation", Nuclear Eng. and Des., 265, pp. 201209, 2013. 
[14] P. Calmon, "Trends and stakes of NDT simulation", J. Nondesctruct. Eval., vol. 31, pp. 339-341, 2012.

[15] M. Augustyniak, Z. Usarek, "Finite element method applied in electromagnetic NDTE: a review", J. Nondesctruct. Eval., vol. 35, n³9, 2016.

[16] A. Skarlatos, T. Theodoulidis, "Study of the non-linear eddy-current response in a ferromagnetic plate: theoretical analysis for the 2D case", NDT \& E Int., Vol. 93, pp. 150-156, 2018.

[17] J. Saitz, "Newton-Raphson method and fixed-point technique in finite element computation of magnetic field problems in media with hysteresis", IEEE Trans. Mag., Vol. 35, Iss. 3, pp. 1398-1401, 1999.

[18] A. Belahcen, A. Arkkio, "A fast fixed-point method for solving magnetic field problems in media of hysteresis", IEEE Trans. Mag., Vol. 44, Iss. 6, pp. 1214-1217, 2008.

[19] M. A. Raulet, B. Ducharne, J.P. Masson, and G. Bayada, "The magnetic field diffusion equation including dynamic hysteresis: a linear formulation of the problem", IEEE Trans. Mag., Vol. 40, $n^{\circ} 2$, pp. 872-875, 2004.

[20] B. Ducharne, G. Sebald, D. Guyomar, G. Litak "Dynamics of magnetic field penetration into soft ferromagnets", Journal of Applied Physics, 243907, pp.1-9, 2015.

[21] A. Yashan, "Measurements and semi-analytical modeling of incremental permeability using eddy current coil in the presence of magnetic hysteresis", in: F. Kojima, T. Takagi, S.S. Udpa, J. Pávó (Eds.), Electromagnetic Nondestructive Evaluation (VI), IOS press, pp. 150-157, 2002.

[22] S. Zhang, B. Ducharne, T. Uchimoto, A. Kita, Y.A. Tene Deffo, "Simulation tool for the Eddy Current Magnetic Signature (EC-MS) non-destructive method, J. of Mag. and Mag. Mat., vol. 513, 167221, 2020.

[23] C.V. Dodd, W.E. Deeds, "Analytical solutions to eddy current probe coil problems", J. App. Phys. 39, (6), pp. 2829-2838, 1968.

[24] C.V. Doff, W.E. Deeds, J.W. Luquire, "Integral solutions to some eddy current problems", Int. J. of NDT 1, pp. 29-90, 1969.

[25] T. P. Theodoulidis, E. E. Kriezis, "Eddy Current Canonical Problems (With Applications to Nondestructive Evaluation)", GA, Norcross: Tech Science Press, 2006.

[26] C.V. Dodd, W.A. Simpson, "Measurement of small magnetic permeability changes by eddy current techniques", Mat. Eval., pp. 217-221, 1971.

[27] C.V. Dodd, "Optimizing defect detection in eddy current testing", Mat. Eval., pp. 59-63, 1971.

[28] C.V. Dodd, W.A. Simpson, "Thickness measurements using eddy current techniques", Mat. Eval., pp. 73-79.

[29] D.C Jiles, D.L Atherton, "Theory of ferromagnetic hysteresis", J. of Mag. and Mag. Mat., Vol. 61, pp. 48-60, 1986.

[30] D.C. Jiles, D.L. Atherton, "Theory of ferromagnetic hysteresis". J. App. Phys. 55, pp. 2115, 1984. 
[31] D.C Jiles, D.L Atherton, "Ferromagnetic hysteresis", IEEE Trans. on Mag.., Vol. 19, pp. 2183-2185, 1983. 